\title{
Modelling diffusion in crystals under high internal stress gradients
}

\author{
David L Olmsted ${ }^{1,4}$, Rob Phillips ${ }^{2}$ and W A Curtin ${ }^{3}$ \\ ${ }^{1}$ Division of Engineering, Brown University, Providence, RI 02912, USA \\ 2 Division of Engineering and Applied Science, California Institute of Technology, Pasadena, \\ CA, USA \\ ${ }^{3}$ Division of Engineering, Brown University, Providence, RI 02912, USA \\ E-mail: olmsted@engin.brown.edu
}

Received 22 July 2003

Published 1 July 2004

Online at stacks.iop.org/MSMSE/12/781

doi:10.1088/0965-0393/12/5/003

\begin{abstract}
Diffusion of vacancies and impurities in metals is important in many processes occurring in structural materials. This diffusion often takes place in the presence of spatially rapidly varying stresses. Diffusion under stress is frequently modelled by local approximations to the vacancy formation and diffusion activation enthalpies which are linear in the stress, in order to account for its dependence on the local stress state and its gradient. Here, more accurate local approximations to the vacancy formation and diffusion activation enthalpies, and the simulation methods needed to implement them, are introduced. The accuracy of both these approximations and the linear approximations are assessed via comparison to full atomistic studies for the problem of vacancies around a Lomer dislocation in Aluminium. Results show that the local and linear approximations for the vacancy formation enthalpy and diffusion activation enthalpy are accurate to within $0.05 \mathrm{eV}$ outside a radius of about $13 \AA$ (local) and $17 \AA$ (linear) from the centre of the dislocation core or, more generally, for a strain gradient of roughly up to $6 \times 10^{6} \mathrm{~m}^{-1}$ and $3 \times 10^{6} \mathrm{~m}^{-1}$, respectively. These results provide a basis for the development of multiscale models of diffusion under highly non-uniform stress.
\end{abstract}

(Some figures in this article are in colour only in the electronic version)

\section{Introduction}

Diffusion of vacancies and impurities in metals is important in the creep, chemical embrittlement, grain boundary segregation, and solute hardening processes, among others, that occur in many structural materials. Because of anisotropic elasticity and/or the presence of defects in

4 Author to whom any correspondence should be addressed. 
materials, such as dislocations, cracks, precipitates, and second-phase particles, this diffusion takes place in the presence of spatially non-uniform, and potentially very large, stresses. Diffusion under uniform stress has been well studied over the years but diffusion in stress fields that vary rapidly over the scale of diffusional hopping distances has been addressed to a much lesser degree [1,2]. Other atomistic studies that consider, for example, solute diffusion near a dislocation, use two-dimensional model crystals which lack the anisotropy that the transition state for diffusion in fcc material possesses [3,4]. Since the continuum stress fields associated with a wide range of defects, particularly dislocations and cracks, are singular in nature, the accurate accounting of diffusion in the stress fields near these singularities may play an important role in establishing the proper dynamics of time-dependent deformation phenomena.

To simulate time-dependent deformation faithfully requires some type of multiscale modelling. Phenomena such as crack growth and solute drag due to dislocation-core/solute interactions require explicit atomistic modelling to properly describe the behaviour in the regions of very high local stresses and stress gradients. Full atomistic resolution via, for instance, molecular dynamics or statics, captures this local behaviour but at the expense of limited sample sizes and the attendant boundary effects that can mask the true behaviour. Thus, higher-level models that involve coarse-grained descriptions of the deformation are needed in regions away from the high stress/stress-gradient regimes so that sufficient sample sizes can be studied. For diffusional processes, the kinetic-Monte-Carlo (kMC) scheme is an efficient approach to tracking material flows over longer time scales than molecular dynamics can support, and it can be combined with continuum diffusion models for regions where the stress gradients are small. The kMC method requires either a previously established database of allowed events and rates or an efficient on-the-fly method for determining those events and rates. For problems of interest here involving non-uniform stress states, the database approach is not generally feasible; although such an approach has been used in the past [1] in a case where a single, static, non-uniform stress state was considered. On-the-fly methods have the advantage of calculating the rates relevant to the particular situations arising during each instant of a simulation but require methods that accurately handle large stresses and stress gradients in an efficient manner. One of the main goals of this work is to develop approximations for on-the-fly calculation of diffusion rates that are accurate in regions of high stress and high stress gradients.

The work here will specifically investigate vacancy diffusion in and around the core of a sessile dislocation. The immobile dislocation provides a high stress and stressgradient environment in which vacancy diffusion takes place, and is thus a convenient test bed for developing various levels of approximation. We consider two appropriate local approximations, requiring the stress tensor and its gradient, within classical rate theory for calculating the diffusion jump rates. These are based on approximations to the various enthalpies associated with the diffusional jump rates, and we compare these results to the enthalpies obtained from full atomistic studies of the problem. The needed enthalpies are the activation enthalpy (the excess enthalpy of the saddle point compared with the bulk material), and the formation enthalpy (the excess enthalpy of a vacancy compared with the bulk material). The saddle point, or transition state, configuration is the configuration of highest enthalpy along the path with the lowest maximum enthalpy between two vacancy configurations. In the case of the fcc crystal, the relevant pairs of vacancy configurations are those where the vacancy in the second configuration is at a nearest neighbour site of the vacancy position in the original configuration.

The first approximation is a 'local' approximation, in which the formation enthalpy for a vacancy at site $\boldsymbol{y}$ and the activation enthalpy of the saddle point configuration on the path between the configuration with a vacancy at $\boldsymbol{y}$ and the configuration with the vacancy at a 
nearest neighbour site $\boldsymbol{y}^{\prime}$ are assumed to be identical to the same enthalpies in a homogeneous crystal under a uniform global applied stress of $\boldsymbol{\sigma}(\boldsymbol{y})$ and $\left[\boldsymbol{\sigma}(\boldsymbol{y})+\boldsymbol{\sigma}\left(\boldsymbol{y}^{\prime}\right)\right] / 2$, respectively. An important subsidiary contribution of the present work is the careful presentation of the simulation methods necessary to implement such an approximation properly. The second approximation is a linear local approximation, where the enthalpy is taken to be a linear function of the stress (as described below), accurate only for small stress. The final approximation is the continuum linear local approximation, where the stress field used in the linear local calculation is that calculated from a continuum model, in this case anisotropic linear elasticity, rather than from direct atomistics. In a multiscale model, the stress will be calculated by coarse-grained or continuum models in some of the regions of space where the kMC models are employed, and hence the continuum linear local approximation is the most desirable from the point of view of practical applications. For the particular problem of vacancy diffusion near a Lomer dislocation studied here, the local approximation is accurate (within $0.05 \mathrm{eV}$ ) to within about $13 \AA$ of the dislocation core, while the linear local approximation is similarly accurate to within about $17 \AA$ of the core, and the continuum linear local approximation is accurate to within about $15 \AA$ of the core. In terms of the gradient of the hydrostatic component of the strain, the local approximation is accurate (within $0.05 \mathrm{eV}$ ) for strain gradients up to roughly $6 \times 10^{6} \mathrm{~m}^{-1}$, and the linear approximation to roughly $3 \times 10^{6} \mathrm{~m}^{-1}$.

As noted earlier, diffusion under stress is not a new topic $[5,6]$. The directly relevant theory was first developed by Dederichs and Schroeder [7], where the transition rate is computed as a function of the defect and saddle point energies and position, the linear approximation is noted, and the diffusion tensor is computed from the transition rates. Recent work extends their analysis to situations with multi-atom unit cells and complex defects [8]. Larche and Cahn [9-11] focused on the stress field created by compositional inhomogeneity that then contributes to the diffusion rates, but the stress is only considered as a driving term (i.e. affecting the defect enthalpy but not the saddle point enthalpy). Full atomistics of vacancy pipe diffusion along a dislocation and an associated kMC database model were developed by Hoagland et al [1], which thus includes all of the inhomogeneities but does not compare their 'exact' results with approximations applicable to multiscale on-the-fly kMC models. Aziz et al [12] have addressed stress effects on diffusion in considerable detail in specific systems but primarily under homogeneous stress states and using the linear approximation. Finally, Laudon et al [2] have modelled diffusion of boron in silicon under non-homogeneous stress using the linear approximation. While the linear approximation has been described and used frequently, we are not aware of any work that tests the limits of its accuracy. Similarly, in recent work describing a non-linear local approximation [8], Daw et al provide no tests of the limits to the accuracy of their approximation. In this paper, we test the accuracy of the linear approximation in regions of high stress gradients by comparing it to atomistic simulations of vacancies and vacancy diffusion saddle points near a dislocation. We also build on much of this prior work to develop a local approximation that improves on the linear approximation and should thus be accurate closer to singular regions, and test it as well as the linear approximation against atomistics to assess their accuracy in some detail. We also discuss in detail the set of simulations required to implement the local model. This study thus provides the groundwork for using atomistic information in multiscale models and gives quantitative guidance on the applicability and accuracy of the local and local linear approximations.

The remainder of this paper is organized as follows. In the next section, we present the basic thermodynamic foundation of transition rate theory. In section 3, we discuss the local and linear approximations, and in section 4 we discuss the accuracy of the approximations for the case of vacancy diffusion near a sessile dislocation, comparing them to atomistic simulations of the full inhomogeneous problem. 


\section{Theory}

We first address the formation of the vacancy state, relative to the crystal without the vacancy. Vacancy formation depends on the difference in thermodynamic potentials of two states: the crystal without vacancies and the crystal with one vacancy. (The crystal may contain fixed sources of internal stress, such as dislocations.) If the external boundary conditions on the material are a uniform hydrostatic pressure then the relevant thermodynamic potential is the enthalpy $H=E+p V$. For an arbitrary uniform external stress state the relevant thermodynamic potential is a generalized enthalpy to be defined shortly, which will also be denoted by $H$.

Let $P_{\mathrm{v}}(\boldsymbol{y})$ be the equilibrium probability that the site $\boldsymbol{y}$ is vacant in the limit of small vacancy concentration. For consistency with the subsequent discussion we include the harmonic approximation to the vibrational entropy in the Gibbs free energy in addition to the ideal entropy of mixing. Minimizing the free energy with respect to concentration at fixed temperature gives

$$
P_{\mathrm{v}}(\boldsymbol{y})=\frac{\prod_{i=1}^{3 n-3} \lambda_{i}^{(\mathrm{b})}}{\prod_{i=1}^{3 n-3} \lambda_{i}^{(\mathrm{v})}(\boldsymbol{y})} \frac{\exp \left[-\beta H_{\mathrm{v}}(\boldsymbol{y})\right]}{\exp \left(-\beta H_{\mathrm{b}}\right)}
$$

where $\left\{\lambda_{i}^{(\mathrm{b})}\right\}$ and $H_{\mathrm{b}}$ are the normal frequencies and enthalpy for the crystal with $n$ atoms and no vacancy (the bulk state) and $\left\{\lambda_{i}^{(\mathrm{v})}(\boldsymbol{y})\right\}$ and $H_{\mathrm{v}}(\boldsymbol{y})$ are those for the crystal with $n$ atoms and one vacancy at position $y$. The frequencies are given by

$$
\lambda_{i}=\frac{1}{2 \pi}\left(\lambda_{i}^{\prime}\right)^{1 / 2}
$$

where the $\lambda_{i}^{\prime}$ are the $3 n-3$ positive eigenvalues of the dynamical matrix

$$
\frac{1}{m} \frac{\partial^{2} E}{\partial \boldsymbol{x} \partial \boldsymbol{x}^{\prime}}
$$

where $m$ is the atomic mass. The bulk and the vacancy states with $n$ atoms each have $3 n-3$ positive eigenvalues, plus three zero eigenvalues corresponding to the centre of mass motion. By defining

$$
v_{\mathrm{b}}=\left(\prod_{i=1}^{3 n-3} \lambda_{i}^{(\mathrm{b})}\right)^{-1}
$$

and $v_{\mathrm{v}}$ in the same way for the vacancy, this can be conveniently written

$$
\begin{aligned}
P_{\mathrm{v}}(\boldsymbol{y}) & =\frac{\nu_{\mathrm{v}}(\boldsymbol{y}) \exp \left[-\beta H_{\mathrm{v}}(\boldsymbol{y})\right]}{\nu_{\mathrm{b}} \exp \left(-\beta H_{\mathrm{b}}\right)} \\
& =\frac{\nu_{\mathrm{v}}(\boldsymbol{y})}{v_{\mathrm{b}}} \exp \left[-\beta \Delta H_{\mathrm{v}}(\boldsymbol{y})\right],
\end{aligned}
$$

where the vacancy formation enthalpy is $\Delta H_{\mathrm{v}}(\boldsymbol{y})=H_{\mathrm{v}}(\boldsymbol{y})-H_{\mathrm{b}}$.

Computing $\Delta H_{\mathrm{v}}(\boldsymbol{y})$ in the presence of a non-uniform stress state is complicated by the fact that the atom removed from the site $y$ is placed at the surface of the material, so that the local stress state at $\boldsymbol{y}$ and the global stress state at the surface must be considered [13]. Considering small hydrostatic stresses for the sake of simplicity (see equations (11) and (37) for the general case), there are three terms that generate the enthalpy change $\Delta H_{\mathrm{v}}(\boldsymbol{y})$ : the change in energy associated with actual bonding in the vacancy region $\Delta E_{\mathrm{v}}(\sigma=0)$; the work gained or lost by moving an atom of volume $v$ from position $\boldsymbol{y}$, at pressure $p(\boldsymbol{y})$, to the surface at the external 
pressure $p^{(\mathrm{e})}$; and the work of inserting the vacancy of $\Delta V_{\mathrm{v}}$ at $\boldsymbol{y}$ against the pressure $p(\boldsymbol{y})$. The sum of these three terms is [13]

$$
\begin{aligned}
\Delta H_{\mathrm{v}}(\boldsymbol{y}) & =\Delta E_{\mathrm{v}}(\boldsymbol{\sigma}=0)+\left[p^{(\mathrm{e})}-p(\boldsymbol{y})\right] v+p(\boldsymbol{y}) \Delta V_{\mathrm{v}} \\
& =\Delta E_{\mathrm{v}}(\boldsymbol{\sigma}=0)+p^{(\mathrm{e})} v-p(\boldsymbol{y})\left(v-\Delta V_{\mathrm{v}}\right)
\end{aligned}
$$

With $\Delta V_{\mathrm{v}}=0.6 v$ for a vacancy in EAM aluminium, it is thus harder to create a vacancy in a crystal under uniform compression $p(y)=p^{(\mathrm{e})}>0$ than in an unstressed crystal, as expected. However, in an unstressed crystal, $p^{(\mathrm{e})}=0$, it is easier to create a vacancy at a point of local compression $p(\boldsymbol{y})>0$ than at a point of local dilatation.

Turning to the issue of diffusion, we wish to compute the jump rate $\Gamma$ at which a vacancy at initial position $\boldsymbol{y}$ moves to a nearest neighbour position $\boldsymbol{y}^{\prime}=\boldsymbol{y}+\boldsymbol{r}$, in the low-concentration limit. This diffusive motion also requires consideration of two states: the state with a vacancy at $\boldsymbol{y}$; and the transition state, which is the saddle point in configuration space between the state with the vacancy at $\boldsymbol{y}$ and the state with the vacancy at $\boldsymbol{y}+\boldsymbol{r}$. Let $H_{\mathrm{t}}$ be the enthalpy of the transition state. Then the jump rate $\Gamma\left(\boldsymbol{y}, \boldsymbol{y}^{\prime}\right)$ is given by [6]

$$
\begin{aligned}
\Gamma\left(\boldsymbol{y}, \boldsymbol{y}^{\prime}\right) & =\frac{v_{\mathrm{t}}\left(\boldsymbol{y}, \boldsymbol{y}^{\prime}\right) \exp \left[-\beta H_{\mathrm{t}}\left(\boldsymbol{y}, \boldsymbol{y}^{\prime}\right)\right]}{v_{\mathrm{v}}(\boldsymbol{y}) \exp \left(-\beta H_{\mathrm{v}}(\boldsymbol{y})\right)} \\
& =\frac{v_{\mathrm{t}}\left(\boldsymbol{y}, \boldsymbol{y}^{\prime}\right)}{v_{\mathrm{v}}(\boldsymbol{y})} \exp \left[-\beta \Delta H_{\mathrm{m}}\left(\boldsymbol{y}, \boldsymbol{y}^{\prime}\right)\right],
\end{aligned}
$$

where the migration enthalpy is $\Delta H_{\mathrm{m}}=\Delta H_{\mathrm{t}}-\Delta H_{\mathrm{v}}$, and, for a solid with $n$ atoms and one vacancy,

$$
v_{\mathrm{t}}=\left(\prod_{i=2}^{3 n-3} \lambda_{i}^{(\mathrm{t})}\right)^{-1}, \quad \lambda_{i}=\frac{1}{2 \pi}\left(\lambda_{i}^{\prime}\right)^{1 / 2}
$$

where the $\lambda_{i}^{\prime}$ are the positive eigenvalues (only $3 n-4$ for the transition state) of the dynamical matrix for the transition state, as defined in equation (3). The notation above explicitly introduces the distinction that the initial vacancy state depends on the position $y$ while the transition state depends on the saddle point configuration along the path from $\boldsymbol{y}$ to $\boldsymbol{y}^{\prime}$. At this stage, $H_{\mathrm{v}}(\boldsymbol{y})$ and $H_{\mathrm{t}}\left(\boldsymbol{y}, \boldsymbol{y}^{\prime}\right)$ are general functionals of the complete deformation state of the system. For future reference, the quantity $\Delta H_{\mathrm{t}}=H_{\mathrm{t}}-H_{\mathrm{b}}$ is termed the activation enthalpy.

Since $v_{\mathrm{t}}\left(\boldsymbol{y}, \boldsymbol{y}^{\prime}\right)$ and $H_{\mathrm{t}}\left(\boldsymbol{y}, \boldsymbol{y}^{\prime}\right)$ are properties of the transition state lying on the path connecting the two equilibrium states $\boldsymbol{y}$ and $\boldsymbol{y}^{\prime}$, they satisfy

$$
\begin{aligned}
& v_{\mathrm{t}}\left(\boldsymbol{y}, \boldsymbol{y}^{\prime}\right)=v_{\mathrm{t}}\left(\boldsymbol{y}^{\prime}, \boldsymbol{y}\right), \\
& H_{\mathrm{t}}\left(\boldsymbol{y}, \boldsymbol{y}^{\prime}\right)=H_{\mathrm{t}}\left(\boldsymbol{y}^{\prime}, \boldsymbol{y}\right) .
\end{aligned}
$$

It is important that any local approximation respect detailed balance, which will be ensured if the flux of vacancies from $\boldsymbol{y}$ to $\boldsymbol{y}^{\prime}$ at equilibrium is equal to the flux in the opposite direction, i.e.

$$
\Gamma\left(\boldsymbol{y}, \boldsymbol{y}^{\prime}\right) P_{\mathrm{v}}(\boldsymbol{y})=\Gamma\left(\boldsymbol{y}^{\prime}, \boldsymbol{y}\right) P_{\mathrm{v}}\left(\boldsymbol{y}^{\prime}\right)
$$

which, from equations (7) and (5) is satisfied if equations (9) are satisfied.

Notice that the enthalpy will always appear as a difference between two states, one with and one without a vacancy, for example. For a large system the strain involved in adding a single vacancy or saddle point defect will be small, and we can define the enthalpy difference as

$$
\Delta H\left(\boldsymbol{y} ;\{\boldsymbol{\sigma}(\boldsymbol{x})\}, \boldsymbol{\sigma}^{(\mathrm{e})}\right)=\Delta E\left(\boldsymbol{y} ;\{\boldsymbol{\sigma}(\boldsymbol{x})\}, \boldsymbol{\sigma}^{(\mathrm{e})}\right)-\boldsymbol{\sigma}_{\alpha \beta}^{(\mathrm{e})} \int_{\partial \Omega} u_{\alpha} \hat{n}_{\beta} \mathrm{d} A,
$$




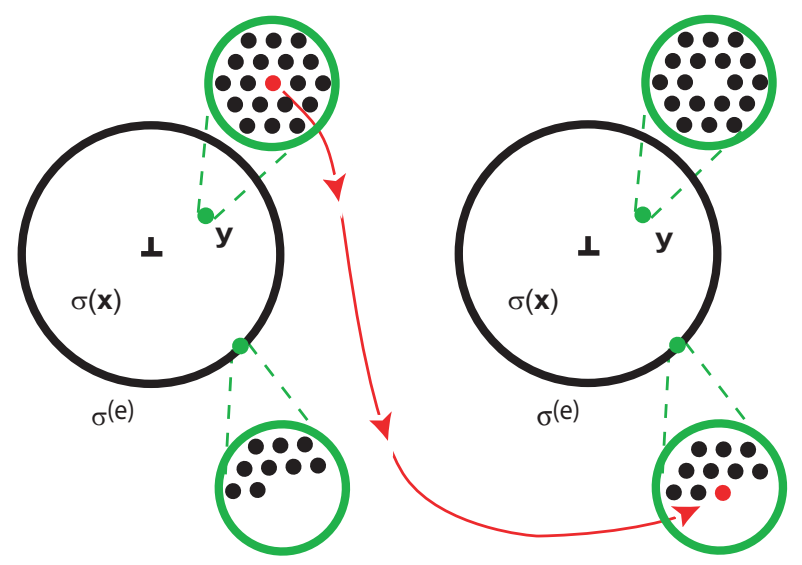

Figure 1. A vacancy is created at a site $y$ in a system under inhomogeneous internal stress, with the atom removed placed at the surface.

where $\boldsymbol{u}$ is the displacement of the boundary on adding the defect at site $\boldsymbol{y}, \hat{\boldsymbol{n}}$ the outward pointing normal unit vector to the surface element $\mathrm{d} A, \partial \Omega$ is the surface of the solid, and $\boldsymbol{\sigma}^{(\mathrm{e})}$ is the external stress. The dependence of the boundary displacements on the stress state, etc., has been suppressed. The atom removed at site $\boldsymbol{y}$ is replaced at the surface in such a way that the surface energy is unchanged. Lower case Greek indices represent spatial coordinates throughout this paper.

\section{Local and linear approximations}

\subsection{Local approximation}

Here we describe an approximation to the above classical rate theory, which we will call the 'local approximation' that avoids the need to compute the enthalpy separately for each vacancy configuration and each transition state configuration of the entire system. Our goal is to compute $\Delta H_{\mathrm{t}}, \Delta H_{\mathrm{v}}, v_{\mathrm{t}}, v_{\mathrm{v}}$, and $v_{\mathrm{b}}$ as simply but as accurately as possible. We expect the enthalpy effects to be more important than the prefactors and so restrict our testing of the local approximation to the enthalpy.

Because creating a vacancy requires putting an atom somewhere, the external stress is involved in the local approximation. A clear presentation of the rationale underlying the local approximation will help clarify the issues (see [13], pp 501-3, where this issue is discussed in the more limited scope of the linear approximation.) We wish to approximate what happens in the system under non-homogeneous stress when a vacancy is created at a point $\boldsymbol{y}$ in the solid, and mass is conserved by placing the extra atom at the surface (in such a way that the surface energy is unchanged) where it experiences the external stress. This is sketched in figure 1 . The enthalpy change in equation (11) consists of the energy change of the system, plus the work done at the boundary against the external stress.

The local approximation is defined by assuming that the enthalpy change for the nonuniform system when the vacancy (or saddle point) is created at site $\boldsymbol{y}$ is equal to the enthalpy change that would occur if an atom were removed from a large block of perfect crystal at the uniform stress $\boldsymbol{\sigma}(\boldsymbol{y})$ so as to create a vacancy (or saddle point) and the atom is then placed in a large block of perfect crystal at uniform stress $\sigma^{(\mathrm{e})}$ in such a way that it remains perfect bulk 


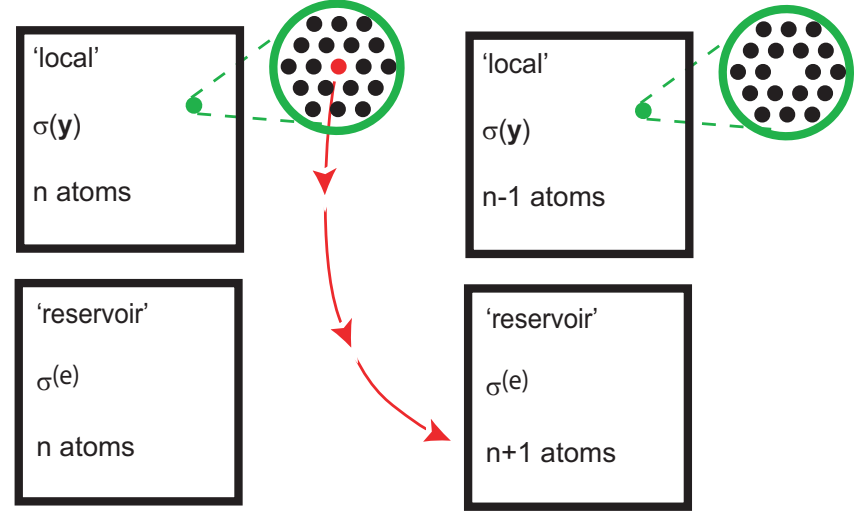

Figure 2. The inhomogeneous system in figure 1 is modelled as a system consisting of two separate blocks of material under uniform stress, a 'local' block at the stress of site $y$ in the inhomogeneous system, and a 'reservoir' block at the external stress. A vacancy is created by removing an atom from the 'local' block and placing it in the 'reservoir' block, which remains defect free.

material. We will denote this as

$$
\Delta H_{\mathrm{d}}\left(\boldsymbol{y} ;\{\boldsymbol{\sigma}(\boldsymbol{x})\}, \boldsymbol{\sigma}^{(\mathrm{e})}\right) \approx \Delta H_{\mathrm{d}}^{(\text {local })}\left[\boldsymbol{\sigma}(\boldsymbol{y}), \boldsymbol{\sigma}^{(\mathrm{e})}\right],
$$

where the defect $\mathrm{d}$ is either $\mathrm{v}$, a vacancy, or $\mathrm{t}$, a saddle point. This process is sketched in figure 2 . Instead of the full non-homogeneous system, we consider a system consisting of two (noninteracting) blocks of material at two different uniform stress states. The local approximation to the enthalpy is then the enthalpy change in this system when the vacancy (or saddle point) is created in the 'local' block at stress $\boldsymbol{\sigma}(\boldsymbol{y})$ and the atom removed is added into the 'reservoir' block at stress $\sigma^{(\mathrm{e})}$. The enthalpy change in this process consists of the energy change in the 'local' block, the energy change in the 'reservoir', the work done at the boundary of the 'local' block against the stress $\boldsymbol{\sigma}(\boldsymbol{y})$, and the work done at the boundary of the 'reservoir' against the stress $\sigma^{(\mathrm{e})}$. Because both blocks are large, and the manipulations involve a single atom, the small relative strain assumption implicit in equation (11) is appropriate.

To compute the local approximation we perform three simulations. Two are simulations of defect-free (bulk) material, one at the external stress $\sigma^{(\mathrm{e})}$ and one at the local stress $\boldsymbol{\sigma}^{(\mathrm{l})}=\boldsymbol{\sigma}(\boldsymbol{y})$; for convenience these each contain the same number of atoms, $n$. The third simulation is of a system with $n-1$ atoms and a defect (vacancy or saddle point) at stress $\sigma^{(1)}$. Each of these simulations provides us with an energy, $E$; a volume, $V$; a shape which we can represent by the average or global deformation gradient, $\boldsymbol{F}$, as measured with respect to a reference state such as the unstressed $n$-atom perfect crystal $^{5}$; and an inverse frequency product $v$ as given by equation (4) or (8). The two bulk simulations therefore provide us with $E_{\mathrm{b}}\left(n, \sigma^{(\mathrm{e})}\right)$, $V_{\mathrm{b}}\left(n, \boldsymbol{\sigma}^{(\mathrm{e})}\right), \boldsymbol{F}_{\mathrm{b}}\left(n, \boldsymbol{\sigma}^{(\mathrm{e})}\right)$, and $\nu_{\mathrm{b}}\left(n, \boldsymbol{\sigma}^{(\mathrm{e})}\right)$, for the system at stress $\boldsymbol{\sigma}^{(\mathrm{e})}$ and similar quantities for the system at stress $\sigma^{(1)}$. The defect simulation provides similar quantities denoted by $E_{\mathrm{d}}\left(n-1, \boldsymbol{\sigma}^{(1)}\right), V_{\mathrm{d}}\left(n-1, \boldsymbol{\sigma}^{(1)}\right), \boldsymbol{F}_{\mathrm{d}}\left(n-1, \boldsymbol{\sigma}^{(1)}\right)$, and $v_{\mathrm{d}}\left(n-1, \boldsymbol{\sigma}^{(1)}\right)$, where $\mathrm{d}$ is either v or $\mathrm{t}$.

The results of a fourth 'simulation', consisting of $n+1$ bulk atoms at stress $\sigma^{(\mathrm{e})}$, are obtained from the bulk simulation of $n$ atoms at stress $\sigma^{(\mathrm{e})}$. Because the $n+1$ atom bulk

5 The average or global deformation gradient is measured by comparing the vectors which specify the periodic boundary conditions of the simulation (relaxed at the given stress) with the vectors which specify the periodic boundary conditions for the reference configuration. 
system is simply a scaled up version of the $n$ atom bulk system, for large $n$

$$
\begin{aligned}
& E_{\mathrm{b}}\left(n+1, \boldsymbol{\sigma}^{(\mathrm{e})}\right)=\frac{n+1}{n} E_{\mathrm{b}}\left(n, \boldsymbol{\sigma}^{(\mathrm{e})}\right), \\
& V_{\mathrm{b}}\left(n+1, \boldsymbol{\sigma}^{(\mathrm{e})}\right)=\frac{n+1}{n} V_{\mathrm{b}}\left(n, \boldsymbol{\sigma}^{(\mathrm{e})}\right), \\
& \boldsymbol{F}_{\mathrm{b}}\left(n+1, \boldsymbol{\sigma}^{(\mathrm{e})}\right)=\left(\frac{n+1}{n}\right)^{1 / 3} \boldsymbol{F}_{\mathrm{b}}\left(n, \boldsymbol{\sigma}^{(\mathrm{e})}\right), \\
& \log \left[\nu_{\mathrm{b}}\left(n+1, \boldsymbol{\sigma}^{(\mathrm{e})}\right)\right]=\frac{n+1}{n} \log \left[\nu_{\mathrm{b}}\left(n, \boldsymbol{\sigma}^{(\mathrm{e})}\right)\right],
\end{aligned}
$$

where $\boldsymbol{F}_{\mathrm{b}}\left(n+1, \boldsymbol{\sigma}^{(\mathrm{e})}\right)$ is measured with respect to the unstressed $n$-atom perfect crystal.

The system consisting of the non-interacting combination of the two bulk simulations, which are relaxed at their respective stresses, is taken as the reference state for calculating the vacancy formation enthalpy and the activation enthalpy. An atom is removed from the $n$-atom simulation at stress $\sigma^{(1)}$, and is nominally added to the $n$-atom simulation at stress $\sigma^{(\mathrm{e})}$. The enthalpy change for the 'two-block' (local plus reservoir) system is then

$$
\begin{aligned}
\Delta H_{\mathrm{d}}^{(\text {local })}= & {\left[E_{\mathrm{d}}\left(n-1, \boldsymbol{\sigma}^{(\mathrm{l})}\right)+E_{\mathrm{b}}\left(n+1, \boldsymbol{\sigma}^{(\mathrm{e})}\right)\right]-\left[E_{\mathrm{b}}\left(n, \boldsymbol{\sigma}^{(\mathrm{l})}\right)+E_{\mathrm{b}}\left(n, \boldsymbol{\sigma}^{(\mathrm{e})}\right)\right] } \\
& -V_{\mathrm{b}}\left(n, \boldsymbol{\sigma}^{(\mathrm{l})}\right) \epsilon_{\mathrm{d}}\left(n, \boldsymbol{\sigma}^{(\mathrm{l})}\right): \boldsymbol{\sigma}^{(\mathrm{l})} \\
& -V_{\mathrm{b}}\left(n, \boldsymbol{\sigma}^{(\mathrm{e})}\right) \epsilon_{\mathrm{b}}\left(n, \boldsymbol{\sigma}^{(\mathrm{e})}\right): \boldsymbol{\sigma}^{(\mathrm{e})}
\end{aligned}
$$

where

$$
\begin{aligned}
& \boldsymbol{\epsilon}_{\mathrm{d}}\left(n, \boldsymbol{\sigma}^{(\mathrm{l})}\right)=\boldsymbol{F}_{\mathrm{d}}\left(n-1, \boldsymbol{\sigma}^{(\mathrm{l})}\right) \boldsymbol{F}_{\mathrm{b}}\left(n, \boldsymbol{\sigma}^{(\mathrm{l})}\right)^{-1}-\mathbf{1}, \\
& \epsilon_{\mathrm{b}}\left(n, \boldsymbol{\sigma}^{(\mathrm{e})}\right)=\boldsymbol{F}_{\mathrm{b}}\left(n+1, \boldsymbol{\sigma}^{(\mathrm{e})}\right) \boldsymbol{F}_{\mathrm{b}}\left(n, \boldsymbol{\sigma}^{(\mathrm{e})}\right)^{-1}-\mathbf{1},
\end{aligned}
$$

and $\boldsymbol{a}: \boldsymbol{b}=\sum_{i j} a_{i j} b_{i j}$. This assumes that $\boldsymbol{\epsilon}_{\mathrm{d}}$ and $\boldsymbol{\epsilon}_{\mathrm{b}}$ are small, but this can always be achieved by choosing $n$ large enough. Furthermore, from equation (15)

$$
\epsilon_{\mathrm{b}}\left(n, \sigma^{(\mathrm{e})}\right)=\frac{1}{3 n} \mathbf{1}
$$

and the local approximation reduces to

$$
\begin{aligned}
\Delta H_{\mathrm{d}}^{(\text {local })}= & E_{\mathrm{d}}\left(n-1, \boldsymbol{\sigma}^{(\mathrm{l})}\right)-E_{\mathrm{b}}\left(n, \boldsymbol{\sigma}^{(\mathrm{l})}\right)+\frac{1}{n} E_{\mathrm{b}}\left(n, \boldsymbol{\sigma}^{(\mathrm{e})}\right) \\
& -V_{\mathrm{b}}\left(n, \boldsymbol{\sigma}^{(\mathrm{l})}\right) \epsilon_{\mathrm{d}}\left(n, \boldsymbol{\sigma}^{(\mathrm{l})}\right): \boldsymbol{\sigma}^{(\mathrm{l})} \\
& -\frac{1}{n} V_{\mathrm{b}}\left(n, \boldsymbol{\sigma}^{(\mathrm{e})}\right) \frac{1}{3} \operatorname{Tr}\left(\boldsymbol{\sigma}^{(\mathrm{e})}\right),
\end{aligned}
$$

which can be computed directly from our three simulations.

The ratio of the $\nu$ s for the 'two-block' system is

$$
\begin{aligned}
\left(\frac{\nu_{\mathrm{d}}}{v_{\mathrm{b}}}\right)^{(\text {local })} & =\frac{\nu_{\mathrm{d}}\left(n-1, \boldsymbol{\sigma}^{(\mathrm{l})}\right) \nu_{\mathrm{b}}\left(n+1, \boldsymbol{\sigma}^{(\mathrm{e})}\right)}{\nu_{\mathrm{b}}\left(n, \boldsymbol{\sigma}^{(\mathrm{l})}\right) \nu_{\mathrm{b}}\left(n, \boldsymbol{\sigma}^{(\mathrm{e})}\right)} \\
& \approx v_{\mathrm{b}}\left(n, \boldsymbol{\sigma}^{(\mathrm{e})}\right)^{1 / n} \frac{\nu_{\mathrm{d}}\left(n-1, \boldsymbol{\sigma}^{(\mathrm{l})}\right)}{\nu_{\mathrm{b}}\left(n, \boldsymbol{\sigma}^{(\mathrm{l})}\right)},
\end{aligned}
$$

but since we have not computed $v$ for any of our large atomistic simulations involving a dislocation, there is nothing to test this local approximation against.

For the formation enthalpy of a vacancy at a site $\boldsymbol{y}$, the local approximation is

$$
\Delta H_{\mathrm{v}}\left(\boldsymbol{y} ;\{\boldsymbol{\sigma}(\boldsymbol{x})\}, \boldsymbol{\sigma}^{(\mathrm{e})}\right) \approx \Delta H_{\mathrm{v}}^{(\text {local })}\left[\boldsymbol{\sigma}(\boldsymbol{y}), \boldsymbol{\sigma}^{(\mathrm{e})}\right] .
$$


Similarly, the local approximation to the activation enthalpy for the saddle point configuration when the vacancy is moving from $\boldsymbol{y}$ to $\boldsymbol{y}^{\prime}$ or vice versa is

$$
\begin{aligned}
& \Delta H_{\mathrm{t}}\left(\boldsymbol{y}, \boldsymbol{y}^{\prime} ;\{\boldsymbol{\sigma}(\boldsymbol{x})\}, \boldsymbol{\sigma}^{(\mathrm{e})}\right) \approx \Delta H_{\mathrm{t}}^{(\text {local })}\left(\boldsymbol{y}^{\prime}-\boldsymbol{y}, \boldsymbol{\sigma}^{(\mathrm{l})}, \boldsymbol{\sigma}^{(\mathrm{e})}\right), \\
& \boldsymbol{\sigma}^{(\mathrm{l})}=\frac{\boldsymbol{\sigma}(\boldsymbol{y})+\boldsymbol{\sigma}\left(\boldsymbol{y}^{\prime}\right)}{2} .
\end{aligned}
$$

Even though the simulation with $n-1$ atoms and a saddle point configuration is at uniform stress $\sigma^{(1)}, \Delta H_{\mathrm{t}}^{(\text {local })}$ depends on the direction of motion $\boldsymbol{y}^{\prime}-\boldsymbol{y}$, since $\boldsymbol{\sigma}^{(\mathrm{l})}$ is not necessarily isotropic. If the stress is approximated from a continuum formula, one might instead use $\sigma\left[\left(\boldsymbol{y}+\boldsymbol{y}^{\prime}\right) / 2\right]$. In any case $\boldsymbol{\sigma}^{(\mathrm{l})}$ needs to be symmetric in $\boldsymbol{y}$ and $\boldsymbol{y}^{\prime}$ in order to satisfy equations (9). Our local approximation for $\Gamma$ is therefore

$$
\Gamma\left(\boldsymbol{y}, \boldsymbol{y}^{\prime}\right) \approx \frac{\left(v_{\mathrm{t}} / v_{\mathrm{b}}\right)^{(\text {local })} \exp \left(-\beta \Delta H_{\mathrm{t}}^{(\text {local })}\right)}{\left(v_{\mathrm{v}} / v_{\mathrm{b}}\right)^{(\text {local })} \exp \left(-\beta \Delta H_{\mathrm{v}}^{(\text {local })}\right)},
$$

where the local approximations for the saddle point in the numerator are evaluated at $\sigma^{(1)}=\left[\sigma(y)+\sigma\left(y^{\prime}\right)\right] / 2, \sigma^{(\mathrm{e})}$, and the direction of motion from $\boldsymbol{y}$ to $\boldsymbol{y}^{\prime}$; and the local approximations for the vacancy in the denominator are evaluated at $\sigma^{(\mathrm{l})}=\boldsymbol{\sigma}(\boldsymbol{y})$ and $\boldsymbol{\sigma}^{(\mathrm{e})}$.

Daw et al [8] describe a local approximation that is similar in some ways to the one we discuss here. Their approximation, however, is not (nor is it intended to be [14]) valid beyond linear order in the stress ${ }^{6}$. Their model does not include any 'remote reservoir' like that in figure 1 in computing the ethalpies relating to vacancy and saddle point formation. What they define, however, are vacancy and saddle point 'creation' volumes and energies, based on comparing a system with $n-1$ atoms and a defect with a system with $n$ atoms [14]. Comparisons of systems with different (fixed) numbers of particles requires justification, and we would argue that the best way to justify such calculations is by invoking a remote reservoir at zero stress.

\subsection{Linear approximation}

In the local approximation, quantities such as $\Delta H_{\mathrm{t}}$ are calculated under conditions of a uniform applied stress. For a general point defect d with $\Delta H_{\mathrm{d}}=H_{\mathrm{d}}-H_{\mathrm{b}}$, the enthalpies can be expanded in a power series in the stress and at small applied stresses we expect the leading linear term to be sufficient. Computing to linear order in $\sigma^{(1)}$ and $\sigma^{(\mathrm{e})}$ in equation (21) we have

$$
\begin{aligned}
& \Delta H_{\mathrm{d}}\left(\boldsymbol{\sigma}^{(\mathrm{l})}, \boldsymbol{\sigma}^{(\mathrm{e})}\right) \approx \Delta H_{\mathrm{d}}(\mathbf{0})-\boldsymbol{\sigma}_{\alpha \beta}^{(\mathrm{l})} \Delta \boldsymbol{V}_{\alpha \beta}^{(\mathrm{d}) *}+v p^{(\mathrm{e})}, \\
& \Delta \boldsymbol{V}^{(\mathrm{d}) *}=V_{\mathrm{b}}(n, \mathbf{0}) \epsilon_{\mathrm{d}}(n, \mathbf{0}),
\end{aligned}
$$

and with $v$ the volume per atom in the bulk at zero stress. $\Delta H_{\mathrm{d}}(\mathbf{0})$ is the energy change when the defect is created in a large system at zero applied stress. For uniform stress, this becomes

$$
\begin{aligned}
& \Delta H_{\mathrm{d}}(\boldsymbol{\sigma}) \approx \Delta H_{\mathrm{d}}(\mathbf{0})-\boldsymbol{\sigma}_{\alpha \beta} \Delta \boldsymbol{V}_{\alpha \beta}^{(\mathrm{d})}, \\
& \Delta \boldsymbol{V}^{(\mathrm{d})}=\Delta \boldsymbol{V}^{(\mathrm{d}) *}+\frac{v}{3} \mathbf{1} .
\end{aligned}
$$

$\Delta \boldsymbol{V}^{(\mathrm{d})}$ is a tensor with units of volume called the strain tensor [12].

We will refer to the 'formation strain tensor', $\Delta \boldsymbol{V}^{(\mathrm{v})}$, in the case where the defect is a vacancy; the 'activation strain tensor', $\Delta \boldsymbol{V}^{(\mathrm{t})}$, when the defect is a saddle point; and to the 'migration strain tensor', $\Delta \boldsymbol{V}^{(\mathrm{m})}$, defined as

$$
\Delta \boldsymbol{V}^{(\mathrm{m})}=\Delta \boldsymbol{V}^{(\mathrm{t})}-\Delta \boldsymbol{V}^{(\mathrm{v})} \text {. }
$$

6 In particular, equations (23) and (24) of [8], while non-linear, are only valid to linear order in the stress, as they overstate effects which are second-order in the stress. 
Equivalently, the linear approximation can be stated in terms of the local strain rather than the local stress [7]. Since $\Delta \boldsymbol{V}^{(\mathrm{t})}$ and $\Delta \boldsymbol{V}^{(\mathrm{v})}$ can be computed by single simulations at zero stress, the linear approximation is clearly more efficient than the full local approximation, which requires similar computations at many stress states. Note that it is possible to continue the expansion in powers of the stress or strain tensor beyond the linear approximation [15], which would provide intermediate approximations between the linear approximation and the full local approximation. Below, we compare the linear approximation to the local approximation and both to the full atomistic results.

\section{Evaluation of local and linear approximations}

\subsection{Simulation details}

The simulations are done using the Ercolessi and Adams [16] glue potential for aluminium. The simulations at a uniform stress or strain are done with periodic boundary conditions in a cell consisting of $4 \times 4 \times 4$ or $6 \times 6 \times 6$ conventional fcc cubic unit cells, and so have 256(864) atoms in the bulk state and 255(863) atoms in both the vacancy and transition state configurations. The shape of the simulation cell at the beginning of a calculation is specified by the periodic boundary vectors $\boldsymbol{P}_{i}, i=1,2,3$. During the simulation the current shape of the cell is specified by the six independent components of a symmetric tensor $\epsilon$, such that the current periodic boundary vectors are

$$
\begin{aligned}
& \boldsymbol{P}_{i \alpha}^{\prime}=\boldsymbol{f}_{\alpha \beta} \boldsymbol{P}_{i \beta}, \quad i=1,2,3, \\
& \boldsymbol{f}_{\alpha \beta}=\delta_{\alpha \beta}+\boldsymbol{\epsilon}_{\alpha \beta} .
\end{aligned}
$$

Limiting $f$ to be symmetric effectively prevents a transition state simulation from rotating in such a way as to change the crystallographic directions of the applied stress.

When performed at constant stress, the simulations minimize the enthalpy

$$
H=E-V_{0} \sigma_{\alpha \beta}^{(\mathrm{e})} \epsilon_{\alpha \beta},
$$

where $V_{0}$ is the volume of the initial simulation cell and $\sigma^{(\mathrm{e})}$ is the external stress. For small $\sigma^{(\mathrm{e})}$, or if the initial cell shape is nearly correct, such a simulation relaxes to a state with Cauchy stress $\sigma^{(\mathrm{e})}$. In general, the simulation has multiple minimization stages, for each of which the initial (reference) cell shape is the relaxed shape of the prior stage. Eventually there is no further change on going one stage further, that is, $\epsilon$ is zero for the final stage, and the Cauchy stress is equal to the desired $\sigma^{(\mathrm{e})}$. For the defect simulations the initial shape of the box is specified as the relaxed shape of the bulk simulation at the same applied stress, which is the reference from which $\epsilon$ for the defect configuration must be measured. Therefore the number of atoms, $n$, must be chosen large enough that $\epsilon$ is small.

For the transition state simulations, the ridge method of Ionova and Carter [17] is used to determine the saddle point.

\subsection{Defect strain tensors}

The measured vacancy formation volume $\Delta V_{\mathrm{v}}$ is $0.595 \Omega$ for the 255 -atom simulation, and $0.596 \Omega$ for the 863 atom simulation, where the atomic volume $\Omega$ is $16.39 \AA^{3}$. Thus, the linear approximation for the vacancy formation enthalpy is

$$
\begin{aligned}
& \Delta H_{\mathrm{v}}\left[\boldsymbol{\sigma}(\boldsymbol{y}), \boldsymbol{\sigma}^{(\mathrm{e})}\right] \approx \Delta H_{\mathrm{v}}(\mathbf{0})-p 6.64 \AA^{3}+p^{(\mathrm{e})} 16.39 \AA^{3}, \\
& p=-\frac{1}{3} \operatorname{Tr}[\boldsymbol{\sigma}(\boldsymbol{y})], \\
& p^{(\mathrm{e})}=-\frac{1}{3} \operatorname{Tr}\left(\boldsymbol{\sigma}^{(\mathrm{e})}\right) .
\end{aligned}
$$




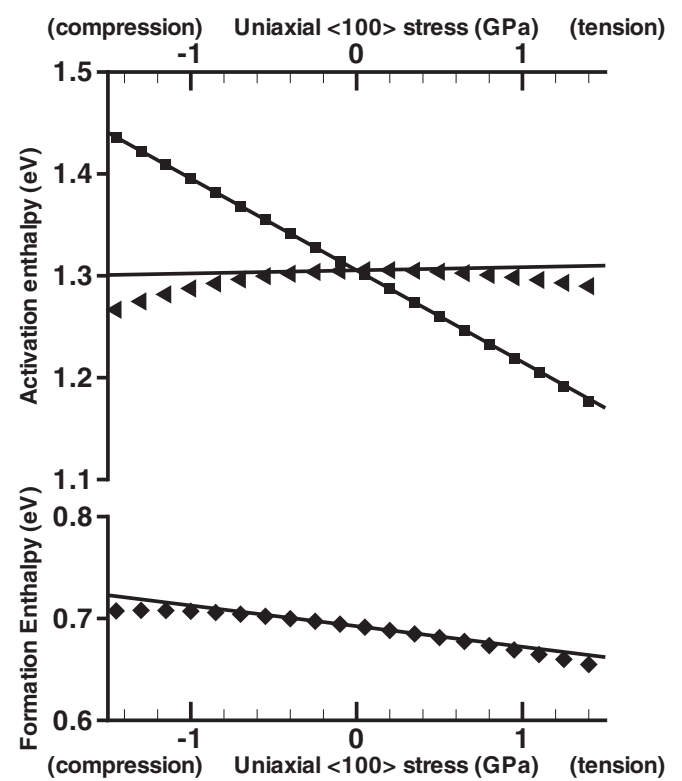

Figure 3. Accuracy of the linear approximation for a case of uniform global stress. Lines indicate the values of the linear approximation, filled symbols the 'exact' results. Diamonds are the vacancy formation enthalpy, while squares and triangles are the activation enthalpies for the two non-equivalent directions of diffusion.

The migration strain tensor for the 255-atom simulation has principal 'strains' of $(0.68,-0.12,-0.35) \Omega$. The positive value is for the $\langle 100\rangle$ direction perpendicular to the jump direction. The $-0.12 \Omega$ is for the jump direction. The value of $-0.35 \Omega$ is for the $\langle 011\rangle$ direction perpendicular to the jump direction. For the 863 atom simulation the migration strain tensor has principal values of $(0.68,-0.12,-0.34) \Omega$. Notice that although the fractional volume increase of $\operatorname{Tr}\left(\Delta \boldsymbol{V}^{(\mathrm{m})}\right)=0.22 \Omega$ on moving the vacancy to the saddle point is only about one-third of the vacancy formation volume, the anisotropy is quite large.

\subsection{Uniform stress}

We first examine a simple case of uniform stress to illustrate the spectrum of behaviour and magnitude of the stress effects, and to test the linear local approximation (our local approximation is exact in this limit). We consider a uniform uniaxial stress along a $\langle 100\rangle$ direction. Figure 3 shows the vacancy formation enthalpy, $\Delta H_{\mathrm{v}}$ and the activation enthalpy, $\Delta H_{\mathrm{t}}$ for this case. When the $\langle 100\rangle$ direction is perpendicular to the motion (4 out of 12 directions of motion) the effect of stress on the activation enthalpy is quite pronounced. For the eight other equivalent directions, the effect is small. However, non-linear effects are more apparent in these latter directions, whereas the linear approximation is quite good for the activation enthalpy for the directions of motion perpendicular to $\langle 100\rangle$ even up to quite large stresses.

\subsection{Lomer dislocation}

To test the local and linear approximations and to investigate a problem of some physical interest, diffusion near a dislocation core in aluminium, we consider the vacancy formation 
and diffusion near a Lomer dislocation, which is a non-glissile edge dislocation. The Lomer dislocation is chosen because it does not disassociate and thus has a single core region and no stacking fault. We anticipate that the local approach, based on a vacancy in a perfect crystal, will break down as the core region is approached because of the large distortions near the core and the changes to the core structure that are induced by a vacancy.

The simulations of the full inhomogeneous system are done in a cylinder with a radius of $50 \AA$, and periodic boundary conditions in the directional parallel to the dislocation. Outside the $50 \AA$ radius, there is an annular region of atoms held fixed at the positions indicated by the anisotropic linear elasticity solution for the dislocation displacement field. The thickness of this region is such that the inner relaxing atoms are effectively in an infinite body. The length in the periodic direction is $28.5 \AA$. Based on calculations at half this length and 1.5 times this length, the effects of the vacancy interacting with its periodic images are negligible at the $28.5 \AA$ separation. The system size is such that the fixed external displacement boundaries accurately mimic the desired state of zero stress at infinity. No change is made in the displacements of the atoms in the fixed region when a vacancy or saddle point is created. The defect enthalpy is then

$$
\begin{gathered}
\Delta H_{\mathrm{d}}^{(\text {dislocation })}\left(\boldsymbol{y}, \boldsymbol{\sigma}^{(\mathrm{e})}=\mathbf{0}\right) \approx E_{\mathrm{d}}^{(\mathrm{dislocation})}\left(m-1, \boldsymbol{y}, \boldsymbol{\sigma}^{(\mathrm{e})}=\mathbf{0}\right)+\frac{1}{n} E_{\mathrm{b}}(n, \boldsymbol{\sigma}=0) \\
-E^{(\mathrm{dislocation})}\left(m, \boldsymbol{y}, \boldsymbol{\sigma}^{(\mathrm{e})}=\mathbf{0}\right)
\end{gathered}
$$

with $m$, the number of atoms, about 23000 . Due to the large system sizes used to avoid boundary effects, no attempt has been made to compute the rate prefactors.

As a reference for understanding the various figures, the atomic positions of the relaxed Lomer dislocation, when viewed along the dislocation line, are shown in figure $4(a)$. The white and grey atoms are in different planes, with an $\mathrm{AB}$ stacking order. The centre of the dislocation is the ring of five-atom columns with empty space shown between; in four of these five columns the atoms are miscoordinated. This core structure predicted by the Ercolessi and Adams potential is in very good agreement with the experimentally determined core structure of the Lomer dislocation in aluminium [18].

In order to apply the local and linear approximations, we need to know the local stresses $\sigma(\boldsymbol{y})$ and $\left[\boldsymbol{\sigma}(\boldsymbol{y})+\sigma\left(\boldsymbol{y}^{\prime}\right)\right] / 2$ to compute the formation and activation enthalpies using equations (24) and (25). Here, we use the stresses at the atomic sites $[19]^{7}$ computed from the dislocation simulation with no vacancy. To indicate the magnitudes of the stresses, figure $4(b)$ shows a contour plot of the pressure field surrounding the dislocation. Pressure is shown because in the linear approximation the vacancy formation enthalpy is linear in the pressure.

Figure 4(c) shows the actual vacancy formation enthalpy around a Lomer dislocation. The stress effects are quite significant. The vacancy formation enthalpy in the unstressed bulk is $\Delta H_{\mathrm{v}}=0.69 \mathrm{eV}$. For a site in the column of atoms just above the centre of the dislocation (as seen in the figure) the vacancy formation enthalpy is $\Delta H_{\mathrm{v}}=0.89 \mathrm{eV}$. Thus, in these locations the equilibrium vacancy concentration at room temperature is reduced by a factor of more than 1000. On the compression side of the dislocation the effect is even stronger. For the two atom columns just below the centre of the dislocation, the vacancy formation enthalpy is only $\Delta H_{\mathrm{v}}=0.04 \mathrm{eV}$. (Larger simulation cells give a value of $0.06 \mathrm{eV}$.)

\footnotetext{
7 Computing the atomistic stress involves dividing by the volume per atom, and so we must define a volume per atom for a single atom near the centre of a dislocation. We have computed the volume per atom for an atom by averaging the distance to its twelve nearest neighbours, to get an average nearest-neighbour distance for the atom. We have then called the volume per atom for an fcc lattice with this nearest-neighbour distance the volume per atom for the atom. A self-consistent measure of the volume, such as a Voronoi construction, where the sum over the atoms of the volume per atom gave the total volume, would be more elegant, but would give similar results for atoms where we would expect the local approximation to be accurate.
} 

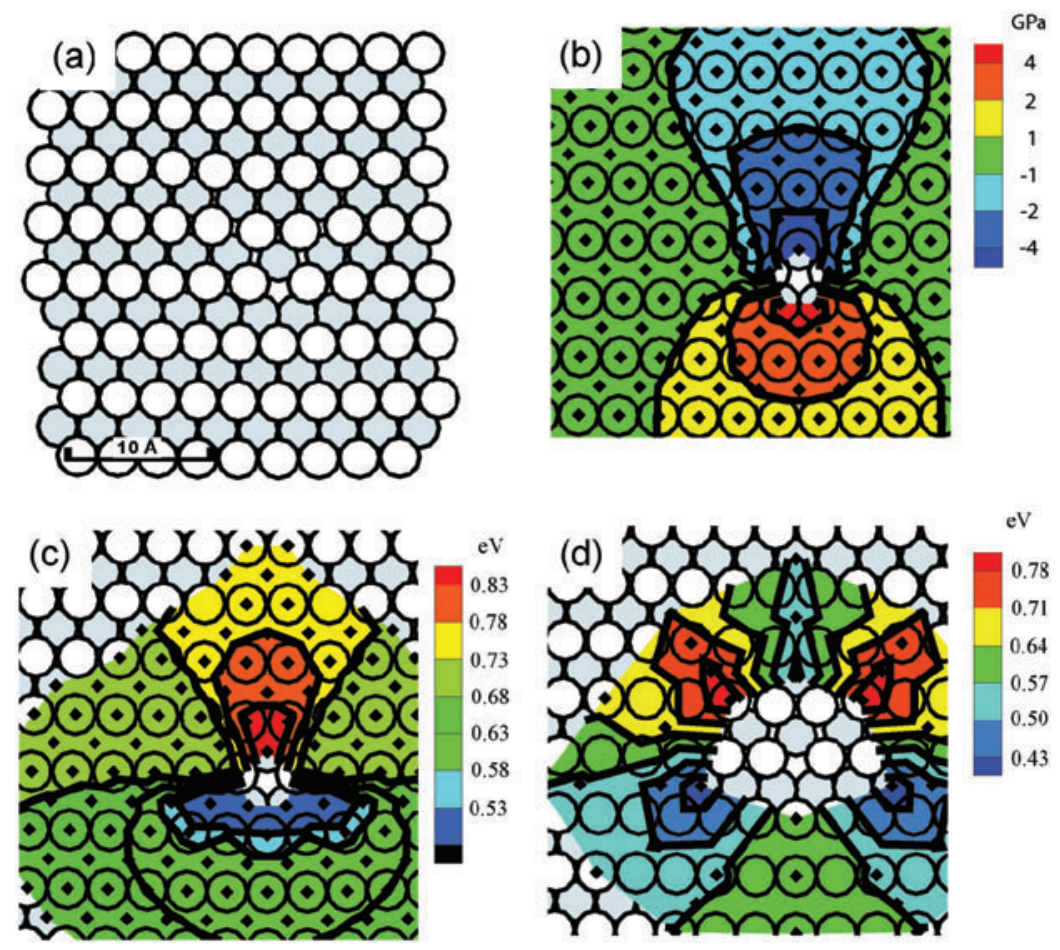

Figure 4. Lomer dislocation in Ercolessi and Adams glue potential for aluminium. (a) Atomic positions near dislocation core. (b) Pressure field of the dislocation in GPa. (c) Vacancy formation enthalpy in $\mathrm{eV}$. The value in bulk material is $0.69 \mathrm{eV}$. (d) Vacancy migration enthalpy for motion parallel to the dislocation line in $\mathrm{eV}$. The value in bulk material is $0.61 \mathrm{eV}$. For the contour plots, data points are marked with black diamonds, and the contours are those generated by the commercial plotting software.

Thus, in equilibrium at room temperature, nearly $10 \%$ of these sites are predicted to be vacant.

We have computed the migration enthalpy for saddle points for about 20 representative sites between 3 and $20 \AA$ from the dislocation core, and each of the seven non-equivalent diffusion directions from those sites. To illustrate a single direction of motion, particularly relevant to pipe diffusion, figure $4(d)$ shows the migration enthalpies for motion parallel to the dislocation line. The migration enthalpies are highly anisotropic. For example, for the sites with the largest and smallest values of the migration enthalpy parallel to the dislocation line, the migration enthalpies for the seven inequivalent directions range as shown in table 1. For comparison, the vacancy migration enthalpy in an unstressed bulk simulation is $0.61 \mathrm{eV}$.

We have computed the vacancy formation enthalpy in the local approximation and in the linear approximation for all nearby atom positions excluding the atoms in the five central columns. The largest error in the local approximation is $0.09 \mathrm{eV}$, and the locations of sites with errors greater than $0.05 \mathrm{eV}$ (equal to $2 k_{\mathrm{B}} T$ at room temperature) are shown in figure $5(a)$. The results for the linear approximation based on the atomistic stress are shown in figure $5(b)$. While not as accurate as the local approximation, errors of more than $0.05 \mathrm{eV}$ are still restricted to the area within about $8 \AA$ of the dislocation centre.

For the local and linear approximations, we have computed the activation enthalpies, rather than the migration enthalpies. The migration enthalpy will generally be accurate wherever the 
Table 1. Anisotropy of migration enthalpy.

\begin{tabular}{lll}
\hline & \multicolumn{2}{l}{ Migration enthalpies for site with } \\
\cline { 2 - 3 } Motion direction & $\begin{array}{l}\text { smallest value } \\
\text { for parallel } \\
\text { direction }\end{array}$ & $\begin{array}{l}\text { largest value } \\
\text { for parallel } \\
\text { direction }\end{array}$ \\
\hline Parallel & 0.39 & 0.83 \\
Perpendicular & $0.29,0.41$ & $0.71,0.70$ \\
Other & $0.56-0.64$ & $0.46-0.48$ \\
\hline
\end{tabular}

activation enthalpy is, and vice-versa, because the approximations to the vacancy formation enthalpy are accurate closer to the dislocation core than these are. For each site, the largest error in the activation enthalpy of the seven possible directions of motion is shown in figure $5(d)$, and the largest errors using the linear approximation in figure 5(e). The error in the local approximation is less than $0.05 \mathrm{eV}$ for sites more than roughly $13 \AA$ from the dislocation centre, and the linear approximation is similarly accurate for sites more than roughly $17 \AA$ from the core.

In performing a higher scale simulation, the atomistic stress would not be available. Rather, stresses from an elasticity solution would be used. We have therefore also computed vacancy formation and activation enthalpies using the continuum linear elastic stress prediction in place of the atomistic stress, in order to test the accuracy of the approximation most likely to be used in practice. In computing the stress field from continuum elasticity, the position of the dislocation is a free parameter which can be chosen to give the best results. Figure 6 shows the anisotropic linear elasticity prediction for the stress field of the dislocation and the values obtained from the atomistic simulation. The results for the vacancy formation enthalpy using the linear approximation with the stress field from continuum linear elasticity are shown in figure $5(c)$. The error is less than $0.05 \mathrm{eV}$ for sites more than roughly $10 \AA$ from the core, slightly worse than for the linear approximation using atomistic stresses. The results for the activation enthalpy using the linear approximation with the continuum linear elastic stress are shown in figure $5(f)$, and the results are similar in accuracy to the linear approximation using the atomistic stresses.

To quantify the dependence of our results in terms of strain, we have computed the gradient of the hydrostatic strain around the Lomer dislocation as follows. For each atom, the nine components of the deformation gradient are fit by least squares to the 36 components of the vectors to its 12 nearest neighbours. (At the core some atoms are miscoordinated, and no calculation is made.) The trace of the Lagrangian strain divided by three is taken as the hydrostatic component of the strain. For atoms for which they and their 12 nearest neighbours all have a successfully calculated hydrostatic strain, we now compute a (three component) gradient as a least squares fit to the 12 differences in hydrostatic strain between the nearest neighbour atoms and the atom. The magnitude of the gradient vector has then contour plotted using commercial plotting software (Tecplot). Examining figure 5 the local and linear approximations are found to be accurate within $0.05 \mathrm{eV}$ for the activation enthalpy for strain gradients up to $6 \times 10^{6} \mathrm{~m}^{-1}$ and $3 \times 10^{6} \mathrm{~m}^{-1}$, respectively.

\section{Conclusions}

We have described a local approximation to the formation and activation enthalpies which govern diffusion in a crystal under highly non-uniform stress. This approximation extends 

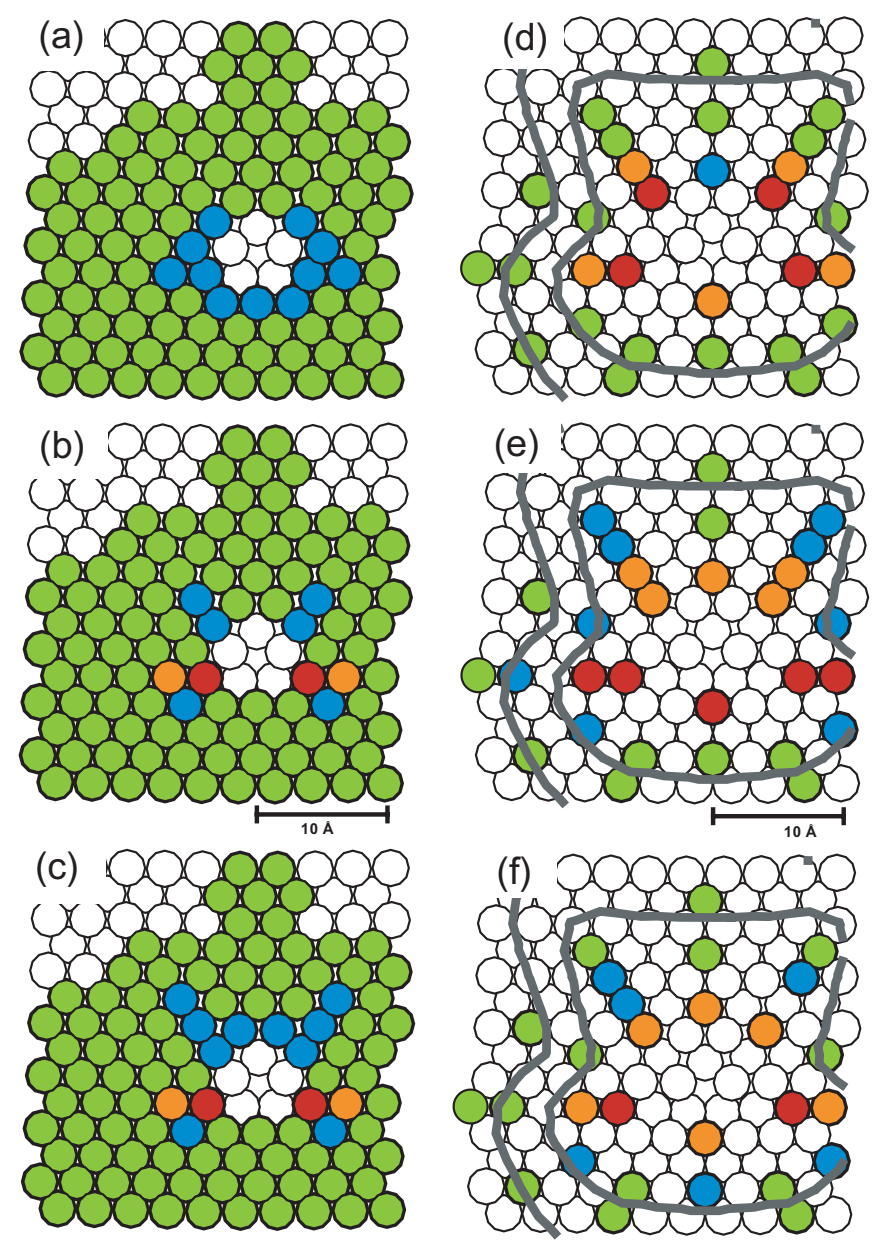

Green: error $<0.05 \mathrm{eV}$.

Blue: $0.05 \mathrm{eV}<$ error $<0.10 \mathrm{eV}$.

Orange: $0.10 \mathrm{eV}<$ error $<0.20 \mathrm{eV}$.

Red: error $>0.20 \mathrm{eV}$.

Figure 5. Accuracy of local and linear approximations as compared to atomistics. Error in: (a) local approximation of the formation enthalpy; $(b)$ and $(c)$ linear approximation of the formation enthalpy, using atomistic and continuum stresses, respectively; $(d)$ local approximation of the activation enthalpy; $(e)$ and $(f)$ linear approximation of the activation enthalpy, using atomistic and continuum stresses, respectively. Coloured sites are those where computations have been made. For each site the activation enthalpy error is the largest error for motion in any direction from the site. The contours shown are for the magnitude of the gradient of the hydrostatic component of the strain of $3 \times 10^{6}$ and $6 \times 10^{6} \mathrm{~m}^{-1}$. The local approximation is accurate within $0.05 \mathrm{eV}$ for sites $1.3 \mathrm{~nm}$ or more from the centre of the dislocation, while the linear approximation is similarly accurate for sites $1.7 \mathrm{~nm}$ or more from the centre.

the previous linear approximation, while still depending only on the local state of stress and its gradient, both of which are required by the linear approximation. While this local approximation will not be accurate in the core of a dislocation, for example, it is accurate closer to the core than the linear approximation. For the particular case of vacancy diffusion near a Lomer dislocation in an EAM model of aluminium, we have tested both the local and linear 


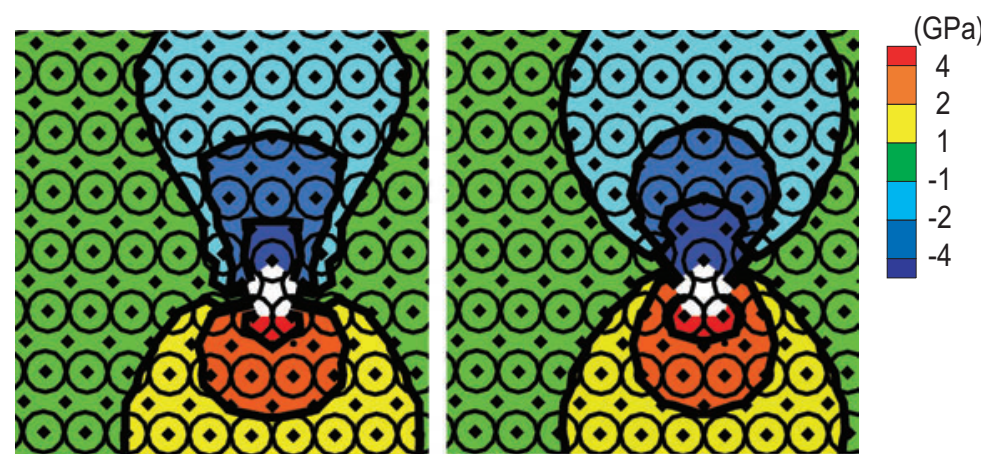

(a)

(b)

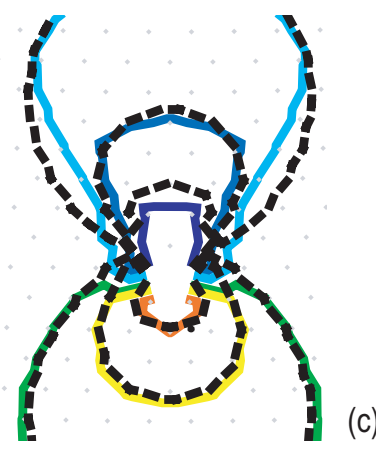

Figure 6. Accuracy of elasticity prediction of pressure field. (a) Pressure field from atomistic stress. (b) Pressure field from anisotropic linear elasticity. (c) The contours from $(a)$ and $(b)$ overlaid. Coloured contours are atomistics, dashed black contours are elasticity.

approximation for the vacancy formation enthalpy and activation enthalpy against atomistic calculations in the presence of the actual dislocation. We find that the local approximation and linear approximations are accurate outside a small region surrounding the core of the dislocation. The cross-sectional area where the local approximation is inaccurate is roughly half that for the linear approximations.

While vacancy diffusion is the simplest situation, this type of local approximation could be extended to interstitial diffusion, and, although it will involve two correlated defects, to diffusion of substitutional impurities via a vacancy mechanism. Furthermore, the formulation developed here, including the detailed simulations needed to create a local model, are applicable for a wide range of problems. The local model developed and tested here and the traditional linear model, which we have also tested, set the stage for multiscale models of diffusion in which the fully atomistically resolved region is reduced to very manageable sizes, which may permit long simulation times and physically meaningful results for diffusion-driven phenomena in solids.

\section{Acknowledgments}

The authors gratefully acknowledge support of this work from the US Air Force Office of Scientific Research through the MURI program 'Virtual Design and Testing of Materials: a Multiscale Approach', grant \#F49620-99-1-0272, and by General Motors through the GM/Brown Collaborative Research Laboratory for Computational Materials Research. The 
authors also thank Laurent Dupuy for helpful comments on a draft of this paper, Professor Emily Carter at UCLA for providing the saddle point code, Stephen M Foiles and Murray S Daw for developing Dynamo, and Professor Ron Miller for modifications to the Dynamo code.

\section{References}

[1] Hoagland R G, Voter A F and Foiles S M 1998 Scr. Mater. 39589

[2] Laudon M, Carlson N N, Masquelier M P, Daw M S and Windl W 2001 Appl. Phys. Lett. 78201

[3] Yoshinaga H and Morozumi S 1971 Phil. Mag. 231367

[4] Wang Y, Srolovitz D J, Rickman J M and Lesar R 2000 Acta Mater. 482163

[5] Koehler J S 1969 Phys. Rev. 1811015

[6] Flynn C P 1972 Point Defects and Diffusion (Oxford: Clarendon)

[7] Dederichs P H and Schroeder K 1978 Phys. Rev. B 172524

[8] Daw M S, Windl W, Carlson N N, Laudon M and Masquelier M P 2001 Phys. Rev. B 64045205

[9] Larché F and Cahn J W 1973 Acta Metall. 211051

[10] Larché F C and Cahn J W 1982 Acta Metall. Mater. 301835

[11] Larché F C and Cahn J W 1985 Acta Metall. 33331

[12] Aziz M J, Sabin P C and Lu G-Q 1991 Phys. Rev. B 449812

[13] Hirth J P and Lothe J 1982 Theory of Dislocations 2nd edn (New York: Wiley)

[14] Daw M S personal correspondence

[15] Woodward C, Rao S I and Dimiduk D M 1998 J. Miner. Met. Mater. Soc. 5037

[16] Ercolessi F and Adams J B 1994 Europhys. Lett. 26583

[17] Ionova I V and Carter E A 1993 J. Chem. Phys. 986377

[18] Mills M J, Daw M S and Foiles S M 1994 Ultramicroscopy 5679

[19] Love A E H 1944 A Treatise on the Mathematical Theory of Elasticity 4th edn (New York: Dover) 